\title{
Factoring und das Kreditwesengesetz
}

Factoring ist kein Bankgeschäft i. S. des $\S 1$ I 2 des Kreditwesengesetzes, sondern eine Finanzdienstleistung gemäß $\S 1$ la 2 Nr. 9 des KWG. Factoringinstitute sind von daher Finanzdienstleistungsinstitute und in der Regel Tochtergesellschaften von Kreditinstituten.

Aufgrund der zentralen Funktion, die Finanzierungsleasing und Factoring bei der Finanzierung der deutschen Industrie spielen, führte auch die Finanzierungsform Factoring zur Aufnahme in den Erlaubniskatalog des KWG. Denn Funktionsstörungen als Folge einer unsoliden Geschäftsführung können schwere Schäden nicht nur im Kundenkreis der betreffenden Unternehmen, sondern auch in weiten Teilen der Wirtschaft verursachen. Diese Gefahr rechtfertigt es, diese Unternehmen einer eingeschränkten Aufsicht zu unterstellen.

Wie bereits ausgeführt sind grundsätzlich zwei Varianten des Factoring zu unterscheiden:

- beim echten Factoring übernimmt der Factor das Zahlungsausfallrisiko der Schuldner.

- $\quad$ beim unechten Factoring behält sich der Factor ein Rückgriffsrecht auf den Forderungs-

verkäufer bei schlechter Bonität oder Zahlungsunfähigkeit des Schuldners vor.

$\S 32$ I Kreditwesengesetz (KWG) enthält die allgemeine Erlaubnispflicht für das gewerbsmäBige Erbringen von Finanzdienstleistungen. Im Erlaubniskatalog ist unter $\S 1$ la $2 \mathrm{Nr}$. $9 \mathrm{KWG}$ das Factoring als der laufende Ankauf von Forderungen auf der Grundlage von Rahmenverträgen mit oder ohne Rückgriff enthalten.

Der Ankauf von Forderungen ist jeder schuldrechtliche Vertrag, der auf den Erwerb einer oder mehrerer Forderungen gerichtet ist. Zivilrechtlich unterliegt nur das echte Factoring dem Kaufrecht. Das unechte Factoring muss dagegen aufgrund des vom Factor nicht übernommenen Bonitätsrisikos dem Darlehensrecht unterworfen werden. Trotzdem erfüllt auch das unechte Factoring eine Finanzierungsfunktion. Daher fällt auch das unechte Factoring unter die Erlaubnispflicht des KWG.

Die Wahrnehmung der Finanzierungsfunktion rechtfertigt es, Factoringunternehmen gleichermaßen unter die Regelung des $\S 1$ la 2 Nr. 9 KWG zu ziehen, ob sie nun neben der Finanzierungsfunktion auch die Delkrederefunktion übernehmen (so genanntes echtes Factoring) oder nicht (so genanntes unechtes Factoring).

Zwischen Factoringkunde und Factor muss eine laufende Geschäftsverbindung bestehen. Der einmalige Kauf einer oder mehrerer Forderungen genügt nicht, um die Genehmigungspflicht auszulösen. Vielmehr muss der Factor dauerhaft und planmäßig dem Kunden Forderungen abkaufen. Daneben muss für die Forderungsverkäufe ein Rahmenvertrag vorliegen. Dabei muss sich zumindest konkludent aus der Geschäftsbeziehung ergeben, dass zukünftig weitere Forderungen vom Factor gekauft werden sollen.

Unternehmen, die mit ihrer Geschäftstätigkeit die Tatbestandsvoraussetzungen des Factoring erfüllen, werden somit Finanzdienstleistungsinstitut gemäß $§ 1$ la $1 \mathrm{KWG}$ und bedürfen daher vor Aufnahme der Geschäftstätigkeit einer Erlaubnis der BaFin.

Ob tatsächlich eine erlaubnispflichtige Geschäftstätigkeit vorliegt oder nicht, entscheidet im Zweifel nach § 4 KWG die Bundesanstalt für Finanzdienstleistungsaufsicht (BaFin). 